\title{
Segurança do paciente na assistência de enfermagem perioperatória e as taxonomias de enfermagem
}

\author{
Patient safety in perioperative nursing care and nursing taxonomies
}

Seguridad del paciente en la atención de enfermería perioperatoria y las taxonomías de enfermería

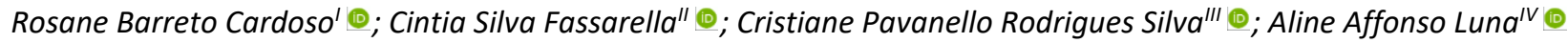 \\ 'Universidade Federal do Rio de Janeiro, Rio de Janeiro, RJ, Brasil; "Universidade do Estado do Rio de Janeiro, Rio de Janeiro, RJ, Brasil; \\ "I'Escola Superior de Saúde de Santa Maria, Porto, Portugal; 'VUniversidade Federal do Estado do Rio de Janeiro, Rio de Janeiro, RJ, Brasil
}

\begin{abstract}
RESUMO
Objetivo: analisar as estratégias da lista de verificação de segurança cirúrgica proposta pela Organização Mundial de Saúde e identificar as taxonomias de enfermagem, a fim de subsidiar um modelo para registro e operacionalização da Sistematização da Assistência de Enfermagem Perioperatória (SAEP). Método: estudo metodológico que visa identificar as ligações das taxonomias de enfermagem, por meio da SAEP. Foram listados os principais termos da lista de verificação de segurança cirúrgica, consultadas as taxonomias de enfermagem, diagnósticos de enfermagem da NANDA-Internacional, classificação dos resultados e das intervenções de enfermagem. Resultados: foi elaborado um modelo para registro e operacionalização da SAEP aplicado a segurança do paciente na assistência de enfermagem perioperatória, conforme cada fase de um fluxo normal de um procedimento cirúrgico. Conclusão: no modelo proposto para SAEP, foram contempladas as taxonomias NANDA-I, NOC e NIC que auxiliarão o enfermeiro no raciocínio clínico para avaliação e implementação de medidas preventivas de incidentes. Descritores: Enfermagem Perioperatória; Processo de Enfermagem; Segurança do Paciente; Terminologia Padronizada em Enfermagem.
\end{abstract}

\begin{abstract}
Objective: to analyze the strategies of the surgical safety checklist proposed by the World Health Organization and identify the nursing taxonomies, as input to a model for recording and operationalizing the Systematization of Perioperative Nursing Care (SAEP). Method: this methodological study aimed to identify the links with nursing taxonomies through the SAEP. The main terms of the surgical safety checklist were listed, and the nursing taxonomies, NANDA-International nursing diagnoses, classification of nursing outcomes and interventions were consulted. Results: a model was developed for recording and operationalizing the SAEP as applied to patient safety in perioperative nursing care, by each phase in a normal surgical procedure flow. Conclusion: the proposed model for SAEP, considered the NANDA-I, NOC, and NIC taxonomies, which will help nurses in clinical reasoning when evaluating and implementing measures to prevent incidents.
\end{abstract}

Descriptors: Perioperative Nursing; Nursing Process; Patient Safety; Standardized Nursing Terminology.

\section{RESUMEN}

Objetivo: analizar las estrategias de la lista de verificación de seguridad quirúrgica propuesta por la Organización Mundial de la Salud e identificar las taxonomías de enfermería, con el fin de subsidiar un modelo de registro y operacionalización de Sistematización de la Atención de Enfermería Perioperatoria (SAEP). Método: estudio metodológico que tiene como objetivo identificar los vínculos de las taxonomías de enfermería a través del SAEP. Se enumeraron los principales términos del checklist de seguridad quirúrgica, consultando las taxonomías de enfermería, diagnósticos de enfermería de NANDA-International, clasificación de los resultados y de las intervenciones de enfermería. Resultados: se desarrolló un modelo de registro y operacionalización del SAEP aplicado a la seguridad del paciente en el cuidado de enfermería perioperatoria, de acuerdo con cada fase del flujo normal de un procedimiento quirúrgico. Conclusión: en el modelo propuesto para SAEP, se consideraron las taxonomías NANDA-I, NOC y NIC, que ayudarán al enfermero en el razonamiento clínico para la evaluación e implementación de medidas preventivas de incidentes.

Descriptores: Enfermería Perioperatoria; Proceso de Enfermería; Seguridad del Paciente; Terminología Normalizada de Enfermería.

\section{INTRODUÇÃO}

A Organização Mundial da Saúde (OMS) define segurança do paciente como redução do risco de danos desnecessários associados à assistência em saúde até um mínimo aceitável ${ }^{1}$. Sabe-se que a segurança do paciente é uma das questões prioritárias da OMS. Em outubro de 2004, a OMS lançou o projeto "Aliança Mundial para a Segurança do Paciente", com o objetivo de despertar a conscientização para a segurança na assistência em saúde 2 .

Frequentemente, a OMS lança desafios globais, em 2009, foi "Cirurgia seguras salvam vidas", dedicado a fundamentos e práticas de segurança cirúrgica, que tem como objetivos aumentar os padrões de qualidade e garantir a segurança nas intervenções cirúrgica, contemplando: prevenção de infecções de sítio cirúrgico; anestesia segura; equipes cirúrgicas seguras e indicadores da assistência cirúrgica ${ }^{3}$. 
Frente a isso, em 2019 o Ministério da Saúde (MS) no Brasil, em parceria com a Organização Pan-Americana da Saúde (OPAS) da OMS lançaram o manual de implementação de medidas para o projeto segurança do paciente: "Cirurgias seguras salvam vidas". Este manual apresenta uma "Lista de verificação de segurança cirúrgica" que foi desenvolvida para ajudar a equipe cirúrgica a reduzir a ocorrência de danos ao paciente ${ }^{3,4}$

Os profissionais de enfermagem destacam-se na equipe cirúrgica na aplicação da lista de verificação de segurança cirúrgica, ao empregar as estratégias e exigências em prol da segurança do paciente ${ }^{5-7}$. Contudo, o enfermeiro precisa estruturar a sua ferramenta metodológica de trabalho, que é o processo de enfermagem (PE), para solidificar e garantir a segurança do paciente na assistência de enfermagem perioperatória, que deverá ser pautada nos preceitos de segurança e qualidade do cuidado.

A Resolução 358/2009 do Conselho Federal de Enfermagem (COFEN) define o PE como um instrumento metodológico que orienta o cuidado de enfermagem, baseado na aplicação prática de teorias de enfermagem, a ser desenvolvido por cinco etapas, a saber: coleta de dados ou histórico de enfermagem; diagnóstico de enfermagem; planejamento da assistência de enfermagem; implementação; e avaliação de enfermagem ${ }^{8}$. O PE viabiliza o raciocínio clínico do enfermeiro, a organização da assistência e a documentação da prática profissional, e por conseguinte um cuidado seguro baseado em evidências ${ }^{9,10}$.

No contexto perioperatório, o PE é denominado Sistematização da Assistência de Enfermagem Perioperatória $(\mathrm{SAEP})^{11}$. A SAEP é um modelo assistencial que foi proposto com a intenção de facilitar a assistência de enfermagem perioperatória, configurando-se como um instrumento que contém informações individuais do paciente, apresentando dados de identificação, anamnese, exame físico, diagnóstico de enfermagem, bem como, intervenções e resultados de enfermagem e avaliação ${ }^{11,12}$

A SAEP é de fundamental importância, uma vez que proporciona uma integração planejada da equipe multidisciplinar de saúde com o paciente/familiares durante todo o processo operatório ${ }^{11,12}$. Em 2002, devido a sua relevância, a SAEP passou a ser uma exigência do COFEN ${ }^{13}$.

Estudos apontam a necessidade de fomentar a segurança do paciente em interface com a SAEP ${ }^{9,10,12,14-18}$. Assim, como utilizar as taxonomias de enfermagem para auxiliar no raciocínio clínico diagnóstico a partir das etapas do $\mathrm{PE}^{9}$.

Diante de tais prerrogativas, este estudo tem como justificativa de analisar as estratégias da lista de verificação de segurança cirúrgica proposta pela OMS e catalogar as taxonomias de enfermagem, a fim de subsidiar um modelo para registro e operacionalização da SAEP que fomente a segurança do paciente no contexto perioperatório. Ainda, pela necessidade de interface dos protocolos de segurança do paciente com o PE, assim como a utilização de taxonomias de enfermagem para auxiliar no raciocínio clínico e registro das ações de enfermagem.

Neste sentido, o objetivo do estudo é analisar as estratégias da lista de verificação de segurança cirúrgica proposta pela OMS, e identificar as taxonomias de enfermagem, a fim de subsidiar um modelo para registro e operacionalização da SAEP que fomente a segurança do paciente.

\section{MÉTOdo}

Estudo metodológico, estruturado em duas partes: 1) Foram analisadas as estratégias da lista de verificação de segurança cirúrgica descrita no manual "Cirurgias seguras salvam vidas", proposta da OMS. A lista de segurança cirúrgica possui três fases, cada uma corresponde a um momento específico no fluxo normal de um procedimento, a saber: o período anterior à indução anestésica (entrada), o período após a indução e antes da incisão cirúrgica (pausa cirúrgica) e o período durante ou imediatamente após o fechamento da ferida, mas anterior à remoção do paciente da sala de operação (saída) ${ }^{3-4}$; 2) Posteriormente, foram consultadas três taxonomias de enfermagem, a saber: a classificação de diagnósticos de enfermagem (DE) da NANDA International (NANDA-I) que organiza conceitos relacionados à respostas humanas reais ou potenciais a condições de saúde/processos de vida, ou uma vulnerabilidade a essa resposta ${ }^{19}$; a classificação dos resultados de enfermagem/Nursing Outcomes Classification (NOC), que reúne uma padronização de resultados sensíveis às intervenções de enfermagem que viabilizam medir o estado clínico do paciente ${ }^{20}$; e a classificação das intervenções de enfermagem/Nursing Interventions Classification (NIC), que agrupa um conjunto de intervenções que consistem em um tratamento baseado no julgamento e conhecimento clínico, no qual o enfermeiro utilizará para elaborar o plano de cuidados ${ }^{21}$

Para identificar as classificações de enfermagem relacionadas às estratégias da lista de verificação de segurança cirúrgica, foram estabelecidas as seguintes etapas: a) Listados os principais termos contidos na lista de verificação de segurança cirúrgica, conforme cada fase do fluxo normal de um procedimento (antes da indução, anestésica; antes da incisão cirúrgica; antes de o paciente sair da sala de operações); b) Mapeado dos possíveis DE conforme os termos previamente listados, consultando a taxonomia NANDA-I (2021-2023); c) Os DE subsidiaram a seleção dos resultados sensíveis às intervenções de enfermagem, com base na ligação NANDA-I/NOC/NIC; d) Seleção das intervenções da NIC com base na 
ligação NANDA-I/NOC/NIC e estratégias estabelecidas na lista de verificação de segurança cirúrgica; e) Por consenso entre os pesquisadores do estudo foram estabelecidos os títulos dos $D E$, resultados e intervenções de enfermagem.

\section{RESULTADOS E DISCUSSÃO}

A SAEP apresenta-se como marco legal ${ }^{8}$ da profissão de enfermagem, pois orienta a organização do cuidado e o registro da documentação da prática profissional, o que torna a assistência de enfermagem mais segura 9,12,14. A SAEP é mais bem conduzida quando associada às taxonomias de enfermagem ${ }^{22,23}$. As taxonomias de enfermagem ou classificações de enfermagem proporcionam benefícios para a prática de enfermagem, condução do raciocínio clínico, qualificação das informações, do registro e da organização da assistência ${ }^{22,23}$.

Deste modo, com base na lista de verificação de segurança cirúrgica proposta pela OMS, foram identificados os elementos da prática de enfermagem (diagnósticos, intervenções e resultados de enfermagem) a partir das taxonomias NANDA-I, NOC, NIC. A fim de elaborar um modelo para o registro e operacionalização da SAEP que possa viabilizar a identificação de riscos e fomentar a segurança do paciente no contexto perioperatório.

A Figura 1 apresenta um modelo para SAEP estruturada mediante as estratégias de segurança do paciente referente a primeira fase de um fluxo normal de um procedimento, conforme a lista de verificação de segurança cirúrgica proposta pela OMS, que corresponde à "antes da indução anestésica".

\begin{tabular}{|c|c|c|}
\hline \multirow{4}{*}{ 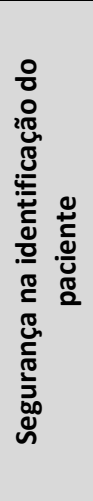 } & NANDA-I & Comunicação verbal prejudicada $(00051)^{19}$ \\
\hline & NOC & $\begin{array}{l}\text { Comunicação (0902) })^{20} \text { Indicadores: Interpretação precisa das mensagens recebidas. } \\
\text { Satisfação do Cliente - comunicação (3002) }{ }^{20} \text { Indicadores: Apresentação da equipe; Métodos alternativos } \\
\text { de comunicação são usados, sempre que necessário. }\end{array}$ \\
\hline & NIC & $\begin{array}{l}\text { Cuidados na admissão }(\mathbf{7 3 1 0})^{21} \text { Tempo e nível de formação necessários para execução: Enfermeiro } \\
\text { Assistencial, } 16-30 \text { minutos } \\
\text { Identificação do paciente }(6574)^{21} \text { Tempo e nível de formação necessários para execução: Enfermeiro } \\
\text { Assistencial, } 15 \text { minutos ou menos }{ }^{21}\end{array}$ \\
\hline & Prescrição & $\begin{array}{l}\text { Apresentar-se e informar seu papel na prestação de cuidados }{ }^{21} \\
\text { Explicar ao paciente a importância da identificação apropriada durante toda sua permanência na } \\
\text { instituição de saúde } \\
\text { Identificar paciente utilizando no mínimo dois identificadores como: nome completo do paciente; nome } \\
\text { completo da mãe do paciente; data de nascimento do paciente; número de prontuário do paciente }\end{array}$ \\
\hline \multirow{4}{*}{ 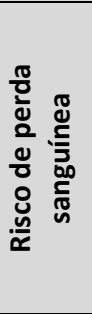 } & NANDA-I & Risco de choque $(00205)^{19}$ \\
\hline & NOC & $\begin{array}{l}\text { Gravidade da perda de sangue }(0413)^{20} \text { Indicadores: Hemoglobina (Hb) diminuída; Hematócritos (Ht) } \\
\text { diminuída; Perda visível de sangue. }\end{array}$ \\
\hline & NIC & $\begin{array}{l}\text { Precauções contra Sangramento }(4010)^{21} \text { Tempo e nível de formação necessários para execução: } \\
\text { Enfermeiro Assistencial, } 31-45 \text { minutos }^{21}\end{array}$ \\
\hline & Prescrição & $\begin{array}{l}\text { Verificar reserva de hemocomponentes ou hemoderivados } \\
\text { Observar os níveis de hemoglobina/hematócrito antes e depois de perda de sangue, conforme indicado } \\
\text { Monitorar os sinais vitais }\end{array}$ \\
\hline \multirow{4}{*}{ 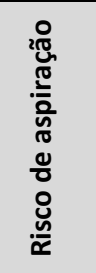 } & NANDA-I & Risco de aspiração (00039) ${ }^{19}$ \\
\hline & NOC & $\begin{array}{l}\text { Prevenção da Aspiração }(\mathbf{1 9 1 8})^{20} \text { Indicadores: Identifica fatores de risco. } \\
\text { Estado Respiratório: permeabilidade das vias aéreas }(\mathbf{0 4 1 0})^{20} \text { Indicadores: Capacidade de expelir } \\
\text { secreções; Frequência respiratória; Tosse. }\end{array}$ \\
\hline & NIC & $\begin{array}{l}\text { Precauções contra Aspiração (3200) }{ }^{21} \text { Tempo e nível de formação necessários para execução: Técnico de } \\
\text { Enfermagem, } 15 \text { minutos ou menos }{ }^{21}\end{array}$ \\
\hline & Prescrição & Manter o equipamento de aspiração disponível ${ }^{21}$ \\
\hline \multirow{4}{*}{ 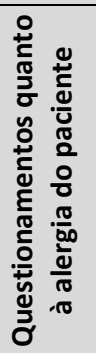 } & NANDA-I & $\begin{array}{l}\text { Risco de reação alérgica }(00217)^{19} \text {; Risco de reação adversa a meio de contraste iodado }(00218)^{19} \text {; Risco } \\
\text { de reação alérgica ao látex }(00042)^{19}\end{array}$ \\
\hline & NOC & $\begin{array}{l}\text { Resposta Alérgica: sistêmica (706) }{ }^{20} \text { Indicadores: Edema de laringe; Urticária; Petéquias; Choque } \\
\text { anafilático. }\end{array}$ \\
\hline & NIC & $\begin{array}{l}\text { Controle de alergias }(6410)^{21} \text { Tempo e nível de formação necessários para execução: Enfermeiro } \\
\text { Assistencial, } 31-45 \text { minutos }^{21}\end{array}$ \\
\hline & Prescrição & $\begin{array}{l}\text { Identificar alergias conhecidas (p. ex, medicamentos, alimentos, insetos, ambientais) e reações usuais }{ }^{21} \\
\text { Colocar pulseira identificadora de alergia no paciente, conforme apropriado } \\
\text { Documentar todas as alergias no prontuário clínico, conforme o protocolo }{ }^{21}\end{array}$ \\
\hline
\end{tabular}

FIGURA 1: Modelo de registro e operacionalização da SAEP a partir das estratégias de segurança do paciente da primeira fase (antes da indução anestésica) da lista de segurança cirúrgica da OMS. Rio de Janeiro, Brasil/Porto, Portugal, 2021. 
Na primeira fase (antes da indução anestésica) da lista de verificação de segurança cirúrgica contém informações relacionadas à: identificação do paciente; demarcação do sítio cirúrgico; anestesia; funcionamento de oxímetro de pulso; questionamentos quanto à alergia do paciente; risco de aspiração/via aérea difícil; e risco de perda sanguínea. Com alicerce nestas estratégias da lista de verificação foram elencados os possíveis DE que poderão elucidar o enfermeiro na estratificação dos riscos e subsidiar a implementação de medidas preventivas.

A identificação correta do paciente e local da intervenção são fundamentais para a segurança cirúrgica, pois os demais processos operatórios são resultantes desta etapa. A demarcação do sítio cirúrgico deverá ser feita preferencialmente com o paciente acordado e consciente, envolvendo o paciente na identificação e na confirmação correta do local da intervenção $0^{3,4}$.

Vale lembrar que nos serviços de saúde a identificação do paciente inicia-se na admissão hospitalar. Deste modo, antes da indução anestésica é importante que o enfermeiro na primeira etapa do PE (coleta de dados), identifique situações que possam colocar em risco o processo de identificação do paciente (como: comunicação verbal prejudicada), além de assegurar que o paciente seja corretamente identificado (utilizar pulseira branca padronizada da instituição com pelo menos dois identificadores: nome completo do paciente; nome completo da mãe do paciente; data de nascimento do paciente; número de prontuário do paciente; e, disposta num membro do paciente para que seja conferido antes do cuidado $)^{4,24,25}$. A comunicação entre o profissional/paciente e profissional/profissional poderá assegurar ao paciente o procedimento correto, prevenindo a ocorrência de erros ${ }^{23}$.

O risco de perda sanguínea deverá ser avaliado antes da indução anestésica, para a implementação de medidas que evitem distúrbios fisiológicos como a hipovolemia transoperatória ${ }^{3}$. Cabe ao enfermeiro identificar DE Risco de choque $(00205)^{19}$, para implementar as intervenções de enfermagem ${ }^{21}$. E durante o procedimento cirúrgico o enfermeiro deverá atentar-se aos sinais e sintomas (taquicardia, hipotensão, oligúria) de choque decorrente de perda sanguínea ${ }^{3}$.

Assegurar as vias aéreas de um paciente submetido à anestesia geral é o momento mais crítico durante a indução anestésica. A incidência de aspiração durante a anestesia geral em cirurgias eletivas foi estimada em 2,6 por 10 mil pacientes $^{3}$. Compete ao enfermeiro avaliar o DE Risco de aspiração (00039) ${ }^{19}$ para instituir as medidas preventivas e minimizar a ocorrência de bronscoaspiração.

Quanto às reações anafiláticas a anestésicos estimam-se a ocorrência de 1:10:000 casos. As causas mais comuns de anafilaxia incluem drogas bloqueadoras neuromusculares, látex, antibióticos, coloides, hipnóticos e opioides ${ }^{3}$. Isto pode ser prevenido pela obtenção de um correto histórico de enfermagem, com identificação do adequada do paciente e documentação no prontuário, assegurando a boa comunicação entre os membros da equipe. A Figura 1 apresenta os possíveis DE e intervenções de enfermagem que poderão ser implementadas pelo enfermeiro, a fim de contribuir com a segurança cirúrgica do paciente.

Já a Figura 2 dispõe um modelo para registro e operacionalização da SAEP estruturada mediante as estratégias de segurança do paciente, na segunda fase da lista de segurança cirúrgica proposta pela OMS que corresponde à "antes da incisão cirúrgica".

As estratégias de segurança da segunda fase (antes da incisão cirúrgica) são referentes à confirmação, quanto à: revisão dos membros da equipe; identificação correta do paciente; sítio cirúrgico correto; elementos críticos de planejamento para a cirurgia (confirmação de utilização de antimicrobianos profiláticos, disponibilização de exames de imagem complementares).

O DE "Risco de lesão por posicionamento perioperatório (00087)" foi aprovado na taxonomia NANDA-I em 1994, antes da publicação do Manual "Cirurgias Seguras Salvam Vidas". Na literatura é evidenciada a aplicação da Escala de Avaliação de Risco para o Desenvolvimento de Lesões Decorrentes do Posicionamento Cirúrgico (ELPO) ${ }^{26}$, que poderá auxiliar o enfermeiro no raciocínio clínico para a identificação deste $\mathrm{DE}$ e viabilizar a implementação de medidas de segurança.

Quanto à infecção que ocorre no paciente cirúrgico no local da operação é conhecida como infecção do sítio cirúrgico (ISC). A ISC contribui para cerca de $20 \%$ de todas as IRAS. As ISC podem aumentar os custos assistenciais, a duração da internação hospitalar de 4-7 dias, além de ocasionar o óbito ${ }^{3}$. Cabe também ao enfermeiro identificar o DE Risco de infecção (00004) ${ }^{21}$ e implementar intervenções preventivas como descritas na Figura 2.

A Figura 3 apresenta um modelo para registro e operacionalização da SAEP que foi estruturado mediante as estratégias de segurança da terceira fase da lista de segurança cirúrgica proposta pela OMS, que corresponde à "antes do paciente sair da sala de cirurgia". 


\begin{tabular}{|c|c|c|}
\hline \multirow{4}{*}{ 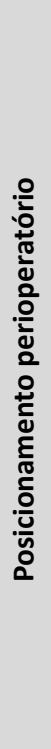 } & NANDA-I & Risco de lesão por posicionamento perioperatório $(00087)^{19}$ \\
\hline & NOC & $\begin{array}{l}\text { Preparo pré-procedimento }(\mathbf{1 9 2 1})^{20} \text {, Indicadores: Conhecimento dos riscos e das complicações } \\
\text { potenciais; Realização da preparação da pele; Participação em lista de verificação pré-procedimento }\end{array}$ \\
\hline & NIC & $\begin{array}{l}\text { Preparo cirúrgico (2930) })^{21} \text { Tempo e nível de formação necessários para execução: Enfermeiro } \\
\text { Assistencial, } 35 \text { minutos }{ }^{21} \\
\text { Precauções cirúrgicas (2920) } \\
\text { Assistencial, mais de } 1 \text { hora }{ }^{21} \\
\text { Posicionamento: intraoperatório }(0842)^{21} \text { Tempo e nível de formação necessários para execução: } \\
\text { Enfermeiro Assistencial, } 35 \text { minutos }{ }^{21}\end{array}$ \\
\hline & Prescrição & $\begin{array}{l}\text { Aplicar lista de verificação de segurança cirúrgica (antes da indução anestésica; antes da incisão } \\
\text { cirúrgica; antes do paciente sair da sala de cirurgia) } \\
\text { Participar do time out pré-operatório, para verificar se o paciente, o procedimento e o sítio estão } \\
\text { corretos }^{21} \\
\text { Aplicar acolchoamento ou evitar pressionar áreas superficiais e acolchoamento sobre proeminências } \\
\text { ósseas }{ }^{21} \\
\text { Aplicar uma faixa de segurança e restrição de membros superiores, quando necessário } \\
\text { Aplicar a escala ELPO26 }\end{array}$ \\
\hline \multirow{4}{*}{ 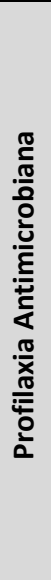 } & NANDA-I & Risco de infecção (00004) ${ }^{19}$; Risco de infecção no sítio cirúrgico $(00266)^{19}$ \\
\hline & NOC & $\begin{array}{l}\text { Controle de Riscos: processo infecioso }(1924)^{20} \text { Indicadores: Identificação de risco de infecção; } \\
\text { Prática de limpeza das mãos }\end{array}$ \\
\hline & NIC & $\begin{array}{l}\text { Controle de infecção (6540) }{ }^{21} \text { Tempo e nível de formação necessários para execução: Enfermeiro } \\
\text { Assistencial, } 35 \text { minutos }{ }^{21} \\
\text { Controle de infecção: transoperatória (6545) }{ }^{21} \text { Tempo e nível de formação necessários para } \\
\text { execução: Enfermeiro Assistencial, mais de } 1 \text { hora } \\
\text { Proteção contra infecção }(6550){ }^{21} \text { Tempo e nível de formação necessários para execução: } \\
\text { Enfermeiro Assistencial, } 35 \text { minutos }{ }^{21}\end{array}$ \\
\hline & Prescrição & $\begin{array}{l}\text { Trocar os locais de linhas centrais e EV periféricas e curativos de acordo com o protocolo } \\
\text { institucional }{ }^{21} \\
\text { Inspecionar a pele e membranas mucosas para rubor, calor extremo e drenagem }{ }^{21} \\
\text { Inspecionar condição de incisão cirúrgica }{ }^{21}\end{array}$ \\
\hline
\end{tabular}

FIGURA 2: Modelo de registro e operacionalização da SAEP a partir das estratégias de segurança do paciente da segunda fase (antes da incisão cirúrgica) da lista de segurança cirúrgica da OMS. Rio de Janeiro, Brasil/Porto, Portugal, 2021.

As estratégias de segurança previstas na terceira fase (antes do paciente sair da sala de cirurgia) são referentes ao registro e revisão do procedimento realizado, tais como: contagem de compressas e instrumentais; identificação de qualquer amostra cirúrgica obtida; revisão de funcionamento de equipamentos e preocupações a respeito da abordagem pós-operatória e da recuperação antes de retirar o paciente da sala cirúrgica.

Antes de o paciente sair da sala de cirurgia é fundamental que a equipe cirúrgica, incluindo o enfermeiro pertencente desta equipe, revise questões anestésicas ou transoperatórias que possam interferir na recuperação pósoperatória do paciente ${ }^{3}$. O objetivo desta etapa da assistência perioperatória é a transferência eficiente e adequada de informações críticas para o planejamento do plano de cuidados no pós-operatório do paciente ${ }^{3}$.

Diante disto é importante que o enfermeiro durante a realização da SAEP identifique o como o DE "Risco de hipotermia perioperatória $(00254)^{21 "}$, que poderá auxiliar na implementação das ações de enfermagem que favoreçam na recuperação pós-operatório do paciente. A hipotermia pode aumentar o risco de infecção e causar problemas de hipocoagulação ${ }^{3}$. O quadro 3 apresenta algumas intervenções de enfermagem que poderão ser implementadas para minimizar os riscos.

O DE "Risco de recuperação cirúrgica retardada" foi aprovado na taxonomia NANDA-I em 2013. Este DE poderá ser identificado pelo enfermeiro ainda na sala de cirurgia. Pois, este DE apresenta como condições associadas: diabetes melito; escore de classificação do estado físico da American Society of Anesthesiologists (ASA) $\geq 3$; procedimento cirúrgico extenso; procedimento cirúrgico prolongado. As condições associadas são indicadores diagnósticos (características definidoras, fatores relacionados, fatores de risco, condições associadas e populações de risco) utilizados para identificar e diferenciar os $\mathrm{DE}^{19}$. 


\begin{tabular}{|c|c|c|}
\hline \multirow{4}{*}{ 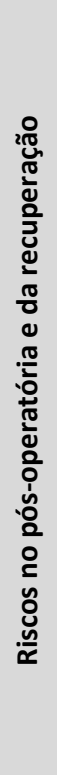 } & NANDA-I & Risco de hipotermia perioperatória $(00254)^{19}$; Risco de recuperação cirúrgica retardada $(00246)^{19}$ \\
\hline & NOC & $\begin{array}{l}\text { Recuperação pós-procedimento (2303) }{ }^{20} \text { Indicadores: Vias aéreas pérvias; Respiração espontânea; } \\
\text { Escore de Aldrete; Termorregulação }\end{array}$ \\
\hline & NIC & $\begin{array}{l}\text { Regulação da temperatura: perioperatória (3902)21 Tempo e nível de formação necessários para } \\
\text { execução: Enfermeiro Assistencial, mais de } 1 \text { hora }{ }^{21} \\
\text { Cuidados pós-anestesia (2870) } \\
\text { Assistencial, } 35 \text { minutos }{ }^{21}\end{array}$ \\
\hline & Prescrição & $\begin{array}{l}\text { Minimizar a exposição do paciente durante o preparo cirúrgico e o procedimento, quando possível }{ }^{21} \\
\text { Monitorar sinais vitais, incluindo temperatura corporal central }{ }^{21} \\
\text { Certificar-se de que o equipamento de aquecimento ativo e os suprimentos estão no lugar e em bom } \\
\text { estado de funcionamento }{ }^{21} \\
\text { Garantir temperatura corporal adequada até o paciente estar acordado e alerta }{ }^{21} \\
\text { Transportar o paciente usando o dispositivo de aquecimento, conforme apropriado }{ }^{21} \\
\text { Monitorar a oxigenação } \\
\text { Monitorar o sítio cirúrgico } \\
\text { Monitorar o retorno da função sensitiva e motora }{ }^{21} \\
\text { Fornecer o prontuário do paciente para a unidade de cuidados pós-operatórios }{ }^{21}\end{array}$ \\
\hline
\end{tabular}

FIGURA 3: Modelo de registro e operacionalização da SAEP a partir das estratégias de segurança do paciente da terceira fase (antes do paciente sair da sala de cirurgias) da lista de segurança cirúrgica seguras proposta pela OMS. Rio de Janeiro, Brasil/Porto, Portugal, 2021.

Para além dos DE utilizados na elaboração do modelo de registro e operacionalização da SAEP fomentado para a promoção da segurança do paciente, na taxonomia NANDA-I no domínio 11 (segurança/proteção) estão dispostos DE relacionados à condição de "estar livre de perigo, lesão física ou dano ao sistema imunológico; preservação contra perdas; proteção da segurança e da ausência de perigos" 19:160, que também poderão ser consultados pelo enfermeiro para avaliar o paciente e traçar medidas preventivas. Após a identificação dos DE, as taxonomias NOC e NIC podem orientar o enfermeiro na implementação e avaliação do plano de cuidados.

É importante mencionar que a NIC ${ }^{21}$ possui uma lista de tempo estimado e nível de formação do profissional necessários para realização de cada intervenção de enfermagem descritas na taxonomia, auxiliando também o enfermeiro no dimensionamento adequado da equipe de enfermagem.

Supõe-se que o modelo elaborado apresenta elementos da prática de enfermagem (DE, intervenções e resultados de enfermagem) que são essenciais para a implementação da SAEP que fomente a segurança do paciente. O enfermeiro poderá consultar as taxonomias sugeridas para complementar o seu plano de cuidados conforme as necessidades de cada paciente. Este modelo também poderá oferecer subsídios para projetos de implementação de sistemas informatizados, sendo utilizado como base para os cadastros da SAEP em prontuários eletrônicos. Assim, como este estudo poderá ser utilizado no processo de ensino e aprendizagem de alunos de graduação de enfermagem, no qual poderá proporcionar o raciocínio clínico para implementação das medidas preventivas, a partir dos elementos da prática de enfermagem.

Uma das limitações do estudo foi testar o modelo proposto de registro e operacionalização da SAEP fomentado para a segurança do paciente cirúrgico no campo empírico. Contudo, o propósito inicial desta pesquisa foi apresentar a possibilidade de integração das estratégias de segurança da lista de verificação de segurança cirúrgica com a operacionalização da SAEP utilizando as taxonomias de enfermagem. O que poderá possibilitar a condução do enfermeiro na implementação de plano de cuidados com ações voltadas a promoção da segurança do paciente cirúrgico em consonância com os protocolos de segurança do paciente estabelecidos pela OMS.

\section{CONCLUSÃO}

Foi possível listar os principais termos contidos na lista de verificação de segurança cirúrgica proposta pela OMS e identificar as taxonomias de enfermagem a partir das ligações NANDA-I, NOC e NIC. Sendo possível propor um modelo que poderá auxiliar o enfermeiro no registro e operacionalização da SAEP alicerçada nas estratégias de segurança do paciente. No modelo proposto para SAEP, foram contempladas as taxonomias NANDA-I, NOC e NIC que poderão auxiliar o enfermeiro no raciocínio clínico para avaliação dos riscos e implementação de medidas preventivas para segurança do paciente no contexto perioperatório. Além de possibilitar o registro adequado da prática profissional e fomentar a segurança do paciente cirúrgico. 


\section{REFERÊNCIAS}

1. World Health Organization. Marco Conceptual de la Clasificación Internacional para la Seguridad del Paciente. Versión 1.1. Informe Técnico Definitivo. Geneva: WHO, 2009. [cited 2021 Sep 10]. Available from:

http://www.who.int/patientsafety/implementation/icps/icps_full_report_es.pdf.

2. World Health Organization. World Alliance for Patient Safety. Sumary of the evidence on patient safety: implications for research. Geneva; 2008. [cited 2021 Sep 10]. Available from: https://apps.who.int/iris/bitstream/handle/10665/43874/9789241596541_eng.pdf?sequence=1\&isAllowed=y.

3. Organização Mundial da Saúde (OMS). Segundo desafio global para a segurança do paciente: cirurgias seguras salvam vidas (orientações para cirurgia segura da OMS) 2009. [cited 2021 Sep 10]. Available from: http://bvsms.saude.gov.br/bvs/publicacoes/seguranca_paciente_cirurgias_seguras_salvam_vidas.pdf.

4. Ministério da Saúde (Br). Agência Nacional de Vigilância Sanitária. Fundação Oswaldo Cruz. Programa Nacional de Segurança do Paciente (PNSP). Protocolo para cirurgia segura. Brasília (DF): Ministério da Saúde; 2013. [cited 2021 Sep 10]. Available from: https://bvsms.saude.gov.br/bvs/saudelegis/gm/2013/prt0529_01_04_2013.html.

5. Botelho ARM, Soares CC, Rodrigues EQ, Santos ELF, Cabral C, Bisagni C, Jorge KM. A atuação do enfermeiro na segurança do paciente em centro cirúrgico de acordo com os protocolos de cirurgia segura e segurança do paciente. Revista Presença [Internet]. 2018 [cited 2021 Sep 10]; 3(10):1-28. Available from: http://sistema.celsolisboa.edu.br/ojs/index.php/numerohum/article/view/138/113.

6. Gutierres LS, Santos JLG, Peiter CC, Menegon FHA, Sebold LF, Erdmann AL. Good practices for patient safety in the operating room: nurses' recommendations. Rev. Bras. Enferm. [Internet], 2018 [cited 2021 Sep 14]; 71 Suppl 6:2940-7. DOI: https://doi.org/10.1590/0034-7167-2018-0449.

7. Criado JVN, Dias BF, Carmo TG. The implementation process of the safe surgery protocolo. Rev. Cuba Enferm. [Internet]. 2017 [cited 2021 Sep 14]; 33(1). Available from: http://www.revenfermeria.sld.cu/index.php/enf/article/view/1028/240.

8. Conselho Federal de Enfermagem. Resolução № 358 do Conselho Federal de Enfermagem, de 15 de outubro de 2009. Brasília; 2009. [cited 2021 Sep 10]. Available from: http://www.cofen.gov.br/resoluo-cofen-3582109_4384.html.

9. Adamy EK, Metelski FK, Argenta C, Silva OM, Zocche DAA. Reflection on the interface between patient safety and the nursing process. Rev. enferm. atenção saúde. [Internet]. 2018 [cited 2021 sep 14]; 7(1): 272-8. Available from: https://pesquisa.bvsalud.org/portal/resource/pt/biblio-912725.

10. Pereira GN, Abreu RNDC, Bonfim IM, Rodrigues AMU, Monteiro LB, Sobrinho JM. Relationship between systematization of nursing care and patient safety. Enferm. Foco [Internet]. 2017 [cited 2021 Aug 21]; 8(2):21-5. Available from: http://revista.cofen.gov.br/index.php/enfermagem/article/view/985.

11. Fengler FC, Medeiro CRG. Nursing care systematization in the perioperative period: analysis of records. Rev. SOBECC. 2020 [cited 2021 Sep 10]; 25(1):50-7. DOI: https://doi.org/10.5327/Z1414-4425202000010008.

12. Santo IMB do E, Matos J da C, Silva CJ da, Almeida R dos P, Santos JLP dos, Silva SM da, Caetano E dos R, Lima RD, Nunes KS, Barbosa S da S. Sistematização da Assistência de Enfermagem Perioperatória (SAEP): Reflexos da Aplicabilidade no Processo de Cuidar. REAS [Internet]. 2020 [cited 2021 Sep 20]; (43):e2945. DOI: https://doi.org/10.25248/reas.e2945.2020.

13. Conselho Federal de Enfermagem - COFEn. Resolução COFEN n. 272, de agosto de 2002: dispõe sobre a Sistematização da Assistência de Enfermagem nas Instituições de Saúde Brasileiras [Internet]. Rio de Janeiro; 2002 [cited 2016 Jun 12]. Available from: http://novo.portalcofen.gov.br/resoluo-cofen-2722102-revogada-pela-resoluao-cofen-n-3582109_4309.html.

14. Riegel F, Oliveira Junior NJ. Nursing process: implications for the safety of surgical patients. Cogitare Enferm. [Internet]. 2017 [cited 2021 Sep 20]; 22(4):1-5. DOI: http://dx.doi.org/10.5380/ce.v22i1.45577.

15. Ribeiro HCTC, Quites HFO, Bredes AC, Sousa KAS, Alves M. Adherence to completion of the safe surgery checklist. Cad. Saúde Pública [Internet]. 2017 [cited 2021 Sep 10]; 33(10):e00046216. DOI: https://doi.org/10.1590/0102-311X00046216.

16. Ribeiro E, Ferraz KMC, Duran ECM. Actitudes de los enfermeros del centro quirúrgico en la sistematización de la asistencia de enfermería perioperatoria. Rev. SOBECC. [Internet]. 2017 [cited 2021 Sep 15]; 22(4):211-7. DOI: https://doi.org/10.5327/Z14144425211700040005.

17. Oliveira MCB, Korb A, Zocche DAA, Bezerra DC, Pertille F, Frigo J. Adhesión del checklist quirúrgico a la luz de la cultura de seguridad del paciente. Rev. SOBECC. [Internet]. 2018 [cited 2021 Sep 15]; 23(1):36-42. DOI: https://doi.org/10.5327/Z14144425212100010007.

18. Souza HX, Sacramento ABAA, Dantas LV, Paranaguá TTB. Surgical patients' perception of safety and their involvement in health care. Rev enferm UERJ. [Internet]. 2020 [cited 2021 Sep 15]; 28:e51948. DOI: http://dx.doi.org/10.12957/reuerj.2020.51948.

19. Herdman STH, Kamitsuru S, Lopes CT. NANDA International Nursing Diagnoses: Definitions and Classification 2021-2023. Twelfth Edition, 2021.

20. Moorhead S, Johnson M, Maas ML, Swanson E. Classificação dos Resultados de Enfermagem (NOC). 6 ed. Rio de Janeiro, Guanabara Koogan, 2020. 608 p.

21. Bulechek GM, Butcher HK, Dochterman JM, Wagner C. Classificação das Intervenções de Enfermagem (NIC). 7 ed. Rio de Janeiro, Guanabara Koogan, 2020. 440p.

22. Larijani TT, Saatchi B. Training of NANDA-I Nursing Diagnoses (NDs), Nursing Interventions Classification (NIC) and Nursing Outcomes Classification (NOC), in Psychiatric Wards: A randomized controlled trial. Nurs Open. [Internet]. 2019 [cited 2021 Aug 23]; 6(2):612-21. DOI: https://doi.org/10.1002/nop2.244. 
23. Carvalho EC. Contribuição da classificação dos resultados de enfermagem na assistência. Arquivos de Ciências da Saúde. [Internet]. 2017 [cited 23 Aug 2021]; 24(1):1-2. Available from:

https://www.cienciasdasaude.famerp.br/index.php/racs/article/view/771/677.

24. Assis TG de, Almeida LF de, Assad LG, Rocha RG, Fassarella CS, Aguiar BGC. Adherence to the correct identification of the patient by hospital wristband. Rev. enferm. UFPE on line. [Internet]. 2018 [cited 2021 Aug 23]; 12(10):2621-7. Available from: https://periodicos.ufpe.br/revistas/revistaenfermagem/article/download/234774/30155.

25. Ministério da Saúde (Br). Agência Nacional de Vigilância Sanitária. Fundação Oswaldo Cruz. Programa Nacional de Segurança do Paciente (PNSP). Anexo 2: Protocolo de identificação do paciente. Brasília: Ministério da Saúde; 2013. Available from: https://bvsms.saude.gov.br/bvs/publicacoes/documento_referencia_programa_nacional_seguranca.pdf.

26. Nascimento FCL, Rodrigues MCS. Risk for surgical positioning injuries: scale validation in a rehabilitation hospital. Rev. LatinoAm. Enfermagem. [Internet]. 2020 [cited 2021 Sep 20]; 28:e3261. DOI: https://doi.org/10.1590/1518-8345.2912.3261. 